\title{
Analyzing Distortion in ASDMs with Loop Delay
}

Amir Babaie-Fishani, Maarten De Bock and Pieter Rombouts

This document is an author's draft version submitted for publication to the 2014 IEEE Int. Symp. Circuits and Syst. (ISCAS 2014).

The actual version was published as [1].

\section{REFERENCES}

[1] A. Babaie-Fishani, M. De Bock, and P. Rombouts, "Analyzing Distortion in ASDMs with Loop Delay," in Proc. IEEE Int. Symp. Circuits and Syst. (ISCAS), 2014, pp. 77-80. 


\title{
Analyzing Distortion in ASDMs with Loop Delay
}

\author{
Amir Babaie-Fishani, Maarten De Bock, and Pieter Rombouts \\ Ghent University (UGent), Dept. ELIS, Sint-Pietersnieuwstraat 41, 9000 Ghent, Belgium \\ Email: Amir.Babaiefishani@elis.ugent.com
}

\begin{abstract}
Recently nearly exact expressions for the distortion in a commonly used family of Pulse Width Modulators (PWMs) known as Asynchronous Sigma Delta Modulators (ASDMs) were presented. Such an ASDM consists of a feedback loop with a schmitt-trigger (or a comparator), and a continuous time loop filter. However these previous results are not yet practically applicable because the effect of unavoidable loop delay (e.g. in the schmitt trigger) was not taken into account. Therefore we now present a more general theory that is also valid when there is a nonzero loop delay. A comparison of the resulting equations with computer simulations demonstrated a very good matching, confirming the validness of the theory. This way, a designer can now easily understand the relationship between the loop filter dynamics and the linearity of an ASDM.
\end{abstract}

\section{INTRODUCTION}

Recently, various applications of Pulse Width Modulators (PWMs) have been presented: e.g. to linearize Analog to Digital converters [1]-[5] or for class-D Power Amplifiers [6], [7]. In these applications, the nonlinearity of the PWM mechanism directly translates into (undesired) overall input-output nonlinearity. Therefore an accurate understanding of the nonlinearity of the PWM mechanism is of direct practical interest. In this paper we study a very popular form of PWM, known as Asynchronous Sigma Delta Modulation (ASDM), which uses a closed loop with a schmitt-trigger (or a comparator) and a continuous time loop filter. We build forth on our previous work [8] where we have already analyzed the distortion for the case without loop delay. The theory in [8] was an improvement to a prior work by Roza [9]. Unfortunately in practice, some loop delay (e.g. due to the comparator) is unavoidable. Moreover in some cases, an explicit delay may even be added intentionally e.g. to control the PWM carrier frequency. Therefore, here the theory of [8] is generalized to cover the case of a loop with delay as well.

\section{PREVIOUS THEORY AND ITS LIMITATIONS}

According to [8], the third harmonic distortion of an ADSM, as in Fig.1 (a), with a loop-filter of $\mathrm{F}(\omega)$ and an input voltage of $v=A \sin (\mu t)$ is (all voltages normalized to \pm 1 ):

$$
d_{3}=\frac{\pi^{2}}{6}\left(\frac{2 A^{2}-8}{-A^{4}}+\frac{6 A^{2}-8}{A^{4} \sqrt{1-A^{2}}}\right) \frac{\operatorname{Re}\left\{F\left(\omega_{c}\right)\right\}}{|F(3 \mu)|}
$$

where $\omega_{c}$ is the self oscillation frequency of the PWM for $v=0$ and $d_{3}$ is the amplitude of the third harmonic tone, $a_{3} \sin (3 \mu t)$, over the amplitude of the main tone, $d_{3}=\frac{a_{3}}{A}$.

This theory was obtained based on some assumptions. First, the highest order of $s^{i}$ in the numerator of $F(s)$ should be exactly one degree less than the denominator to have a stable oscillation. Second, it assumes that all poles and zeros of the

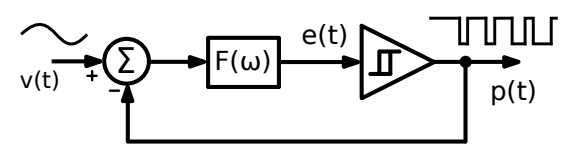

(a)

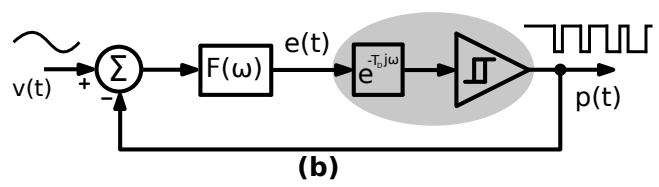

Fig. 1. General representation of an ASDM, (a) without extra delay in the loop, and (b) with loop delay.

filter are at much lower frequency than $\omega_{c}$, and finally, the filter should satisfy the following conditions for $\omega \geqslant \omega_{c}$ :

$$
\operatorname{Re}\{F(\omega)\} \approx \frac{1}{\left(\tau_{r} \cdot \omega\right)^{2}} \quad \text { and, } \operatorname{Im}\{F(\omega)\} \approx \frac{1}{\tau_{i} \cdot \omega}
$$

where $\tau_{r}$ and $\tau_{i}$ are filter dependent constants and are not necessarily positive and/or real. Then, given a schmitt trigger threshold voltage of $\pm V_{t h}$ we will have $\omega_{c}=\frac{\pi}{2 \tau_{i} \cdot V_{t h}}$.

The theory in [8] only applies to a certain category of ASDMs where the schmitt trigger is ideal (has no delay). But in an actual circuit, the schmitt trigger always has a delay, modelled as $T_{D}$ in Fig. 1 (b), and as the oscillation frequency of the PWM increases, the loop delay becomes more important in determining the oscillation frequency and the distortion.

In order to evaluate $d_{3}$ for the case with delay, the first solution that comes to mind is to merge the delay of the schmitt trigger in the loop-filter as a $e^{-T_{D} j \omega}$ factor and use (1) for calculating the third harmonic with the new loop-filter, $G(\omega)=$ $F(\omega) \cdot e^{-T_{D} j \omega}$. But it can be easily shown that $\operatorname{Re}\{G(\omega)\}$ and $\operatorname{Im}\{G(\omega)\}$ will not satisfy the conditions in (2).

\section{PROPOSED APPROACH}

Here we will revisit the equations in [8] with similar assumptions while considering a delay of $T_{D}$ for the schmitt trigger. Bare in mind that delay anywhere in the loop can be modelled this way without affecting the spectral qualities of the signals.

In Fig. $1(\mathrm{~b}), v(t)$ is a band limited input signal, $e(t)$ is the filtered error signal at the input of the schmitt-trigger, and $p(t)$ is the output square wave $( \pm 1)$ with a pulse width of $\alpha$ and duration of $T$. As it will be shown later in this section, in this type of modulation, not only the duty cycle of the output square wave, $\frac{\alpha}{T}$, but also its carrier frequency are dependent on the input signal. The self oscillation frequency of this PWM for a zero input is called $\omega_{c}$ and $\omega_{1}=\frac{2 \pi}{T}$ is the (instantaneous) 


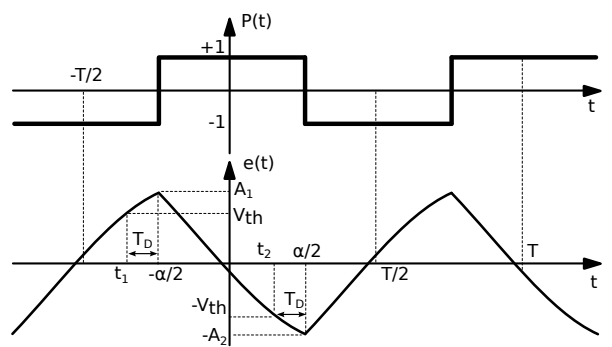

Fig. 2. Typical waveforms of an schmitt trigger based ASDM with delay.

carrier frequency which is a function of the input signal, $v$. According to Fig. 1 (b) we can write the signal at e(t):

$$
e(t)=(v-p(t)) \otimes f
$$

where $f(t)$ is the time domain impulse response of the filter, $F(\omega)$, and $\otimes$ is the convolution.

Assuming that the input signal, $v$, changes at a much slower rate than the carrier frequency, it can be considered approximately constant during each periods of $p(t)$. Then $p(t)$, which is a square wave, can be described by its Fourier series:

$$
p(t)=\left(\frac{2 \alpha}{T}-1\right)+4 \times \operatorname{Re}\left\{\sum_{n=1}^{\infty} \frac{\sin \left(n \omega_{1} \frac{\alpha}{2}\right)}{n \pi} e^{j n \omega_{1} t}\right\}
$$

The $\left(\frac{2 \alpha}{T}-1\right)$ term in $p(t)$, which will be referred to as $u$ hereafter, is the baseband component of $p(t)$ which includes the desired signal and its harmonic distortion components, so we can say $u=\left(\frac{2 \alpha}{T}-1\right)$ or $\omega_{1} \alpha=\pi(u+1)$.

Fig. 2 shows typical waveforms of an ASDM. As the figure shows, the schmitt-trigger toggles with a delay of $T_{D}$ after $e(t)$ reaches its positive or negative thresholds, $V_{t h}$ or $-V_{t h}$. So if we use $t=\frac{\alpha}{2}-T_{D}$ and substitute (4) in (3), we obtain:

$$
\begin{aligned}
-V_{t h}=(v-u) \otimes f-4 \sum_{n=1}^{\infty}\left(\frac{\sin \left(n \omega_{1} \frac{\alpha}{2}\right)}{n \pi}\right) \times \\
\left(\operatorname{Re}\left\{F\left(n \omega_{1}\right)\right\} \cos \left(n \omega_{1}\left(\frac{\alpha}{2}-T_{D}\right)\right)-\right. \\
\left.\operatorname{Im}\left\{F\left(n \omega_{1}\right)\right\} \sin \left(n \omega_{1}\left(\frac{\alpha}{2}-T_{D}\right)\right)\right)
\end{aligned}
$$

and for $t=\frac{-\alpha}{2}-T_{D}$ we will have:

$$
\begin{array}{r}
V_{t h}=(v-u) \otimes f-4 \sum_{n=1}^{\infty}\left(\frac{\sin \left(n \omega_{1} \frac{\alpha}{2}\right)}{n \pi}\right) \times \\
\left(\operatorname{Re}\left\{F\left(n \omega_{1}\right)\right\} \cos \left(n \omega_{1} \frac{\alpha}{2}+n \omega_{1} T_{D}\right)+\right. \\
\left.\operatorname{Im}\left\{F\left(n \omega_{1}\right)\right\} \sin \left(n \omega_{1} \frac{\alpha}{2}+n \omega_{1} T_{D}\right)\right)
\end{array}
$$

By adding the last two equations we will have:

$$
\begin{gathered}
0=(v-u) \otimes f-2 \sum_{n=1}^{\infty} \frac{\sin \left(n \omega_{1} \alpha\right)}{n \pi} \times \\
\left(\operatorname{Im}\left\{F\left(n \omega_{1}\right)\right\} \sin n \omega_{1} T_{D}+\operatorname{Re}\left\{F\left(n \omega_{1}\right)\right\} \cos n \omega_{1} T_{D}\right)
\end{gathered}
$$

and subtracting them will give:

$$
\begin{gathered}
\frac{-\pi V_{t h}}{2}=\sum_{n=1}^{\infty} \frac{1-\cos \left(n \omega_{1} \alpha\right)}{n} \times \\
\left(\operatorname{Im}\left\{F\left(n \omega_{1}\right)\right\} \cos n \omega_{1} T_{D}-\operatorname{Re}\left\{F\left(n \omega_{1}\right)\right\} \sin n \omega_{1} T_{D}\right)
\end{gathered}
$$

If the ASDM has a stable oscillation, then we can deduce from Fig. 2 that $2 T_{D} \leq \alpha+T_{D} \leq T=\frac{\omega_{1}}{2 \pi}$ and from that we can say $0 \leq \omega_{1} \alpha \pm \omega_{1} T_{D} \leq 2 \pi$. The terms $\operatorname{Re}\left\{F\left(n \omega_{1}\right)\right\}$ and $\operatorname{Im}\left\{F\left(n \omega_{1}\right)\right\}$ are obtained from (2). This allows us to solve the series in (8) using [11]. Then (8) will result in:

$$
\omega_{1}=\omega_{c} \cdot\left(1-u^{2}\right)=\frac{\pi\left(\tau_{i} T_{D}-\tau_{r}^{2}\right) \cdot\left(1-u^{2}\right)}{2 \tau_{r}^{2} \tau_{i} V_{t h}+\tau_{i} T_{D}^{2}-2 \tau_{r}^{2} T_{D}}
$$

This equation accurately gives the instantaneous and self oscillation frequencies, $\omega_{1}$ and $\omega_{c}$, as functions of the output base band signal, $u$, and the parameters of the modulator.

Same as above, we can calculate the series in (7) using (2), (9), [11], and the fact that $0 \leq \omega_{1} \alpha \pm \omega_{1} T_{D} \leq 2 \pi$. With that all, we can simplify (7) which will give:

$$
(u-v) \otimes f=\frac{\pi^{2} u}{6\left(\tau_{r} \omega_{c}\right)^{2}\left(1-u^{2}\right)}-\frac{u T_{D}^{2}}{2 \tau_{r}^{2}}+\frac{u T_{D}}{\tau_{i}}
$$

This equation gives an accurate relationship between the input signal, $v$, and the output baseband signal, $u$. In order to avoid the need to solve an implicit equation to obtain $u$ as a function of $v$, we can simplify (10) using Perturbation Theory. So at first we make a rough first order estimation from (10). We can see that the terms on the right side of the equation are much smaller than the ones on the left, so we can say $u \approx v$. By applying this estimation to (10) we will obtain this simplified equation which gives $u$ as an explicit function of $v$ :

$$
(u-v) \otimes f \approx \frac{\pi^{2} v}{6\left(\tau_{r} \omega_{c}\right)^{2}\left(1-v^{2}\right)}-\frac{v T_{D}^{2}}{2 \tau_{r}^{2}}+\frac{v T_{D}}{\tau_{i}}
$$

Now we will apply a sinusoidal input voltage, $v=A \sin \mu t$, to (11) to obtain the harmonic distortions of the ASDM. We can see that the right hand of this equation is an odd function of $v$, so we can expect $u$ to only have odd harmonics of $v$. Therefore, we can replace $u$ with its Fourier expansion:

$$
u=\sum_{n} a_{n} \sin \left(n \mu t-\theta_{n}\right), \quad n=1,3,5, \ldots
$$

Since the terms on the right hand of (11) are much smaller than those on the left, for the main tone we can say $a_{1} \approx A$. In the right hand of (11), only the first term can produce harmonic distortion. So if we write the Fourier expansion on both sides of (11) only for the harmonic tones $(n=3,5, \ldots)$ we will have:

$$
\sum_{n} a_{n} \sin \left(n \mu t-\theta_{n}\right) \otimes f \approx \frac{\pi^{2}}{6 \tau_{r}^{2} \omega_{c}^{2}} \sum_{n} \mathrm{~K}_{n}(A) \sin (n \mu t)
$$

where $\mathrm{K}_{n}$ comes from this Fourier integral:

$$
\mathrm{K}_{n}(A)=\frac{\mu}{\pi} \int_{\frac{-\pi}{\mu}}^{\frac{\pi}{\mu}} \frac{A \sin (\mu t) \cdot \sin (n \mu t)}{1-(A \sin (\mu t))^{2}} d t
$$

and from (13) we can deduce for each harmonic that:

$$
\theta_{n}=\angle[F(n \mu)], \quad a_{n} \cdot|F(n \mu)|=\frac{\pi^{2}}{6 \tau_{r}^{2} \omega_{c}^{2}} \mathrm{~K}_{n}(A)
$$

and by considering (2) for $\omega_{c}$, we can find the ratio of the magnitude of the nth harmonic over the main tone:

$$
d_{n}=\frac{a_{n}}{a_{1}} \approx \frac{a_{n}}{A}=\frac{\pi^{2}}{6} \frac{\mathrm{K}_{n}(A)}{A} \frac{\operatorname{Re}\left\{F\left(\omega_{c}\right)\right\}}{|F(n \mu)|}
$$


The integral in (14) can be solved for any of the harmonics. For $n=3,5$, for example, it gives (for $A \leq 1$ ):

$$
\begin{gathered}
\mathrm{K}_{3}(A)=\frac{2 A^{2}-8}{-A^{3}}+\frac{6 A^{2}-8}{A^{3} \sqrt{1-A^{2}}} \\
\mathrm{~K}_{5}(A)=\frac{2 A^{4}-24 A^{2}+32}{-A^{5}}+\frac{10 A^{4}-40 A^{2}+16}{A^{5} \sqrt{1-A^{2}}}
\end{gathered}
$$

By substituting (17) in (16) we can find the 3rd harmonic distortion of an ASDM with loop delay:

$$
d_{3}=\frac{\pi^{2}}{6}\left(\frac{2 A^{2}-8}{-A^{4}}+\frac{6 A^{2}-8}{A^{4} \sqrt{1-A^{2}}}\right) \frac{\operatorname{Re}\left\{F\left(\omega_{c}\right)\right\}}{|F(3 \mu)|}
$$

and the term $\operatorname{Re}\left\{F\left(\omega_{c}\right)\right\}$ can be calculated using (9) and (2):

$$
\begin{array}{r}
d_{3}=\frac{1}{6}\left(\frac{2 A^{2}-8}{-A^{4}}+\frac{6 A^{2}-8}{A^{4} \sqrt{1-A^{2}}}\right) \frac{1}{|F(3 \mu)|} \times \\
\frac{\left(2 \tau_{r}^{2} \tau_{i} V_{t h}+\tau_{i} T_{D}^{2}-2 \tau_{r}^{2} T_{D}\right)^{2}}{\tau_{r}^{2}\left(\tau_{i} T_{D}-\tau_{r}^{2}\right)^{2}}
\end{array}
$$

In ASDMs without loop delay (assuming that a high order aggressive loop filter is not chosen) the signal at the input of the schmitt trigger is limited to $\pm V_{t h}$, e.g. Fig. 3(a). After introducing delay to the loop, a certain amplitude modulation effect occurs, Fig.3(b). The (above) envelope of this effect can be calculated by evaluating $e\left(t=\frac{-\alpha}{2}\right)$, see Fig. (2). By replacing (4) in (3) and calculating the series we will have:

$$
e\left(\frac{-\alpha}{2}\right)=u T_{D}\left(\frac{T_{D}}{2 \tau_{r}^{2}}-\frac{1}{\tau_{i}}\right)-\frac{T_{D}}{\tau_{i}}+\frac{\tau_{r}^{2} V_{t h}-0.5 T_{D}^{2}}{\tau_{r}^{2}-\tau_{i} T_{D}}
$$

\section{IMPLICATIONS AND RESULTS}

\section{A. ASDM equivalency}

A surprising outcome is that (1) and (19) are the same and that implies that an ASDM with a schmitt trigger threshold voltage of $V_{t h}$ and loop delay of $T_{D}$ is equivalent (regarding its distortion) with another ASDM that has an identical loop filter and identical oscillation frequency with a threshold voltage of $V_{t h, e q}$ and zero delay where $V_{t h, e q}$ can be calculated using (20) by solving $d_{3}\left(V_{t h}, T_{D}\right)=d_{3}\left(V_{t h, e q}, 0\right)$. This value of equivalent threshold happens to be equal to $\left.e\left(\frac{-\alpha}{2}\right)\right|_{u=0}$ in (21).

\section{B. amplitude modulation}

In previous works, the amplitude modulation discussed before (21) was believed to be a source of distortion. In [10] a method was presented to cancel out this effect through an active feedback. The threshold voltage of the schmitt trigger was dynamically reduced to keep the envelope of the signal at the input of the schmitt trigger constant and equal with the desired threshold voltage. In this way, the modulator's waveform and behaviour will be similar to one without delay. This method is only applicable when the schmitt trigger is implemented using op-amp and resistors. However, as it has been shown in this work, the distortion in an ASDM with delay is the same as an equivalent one without delay and there is no need for extra circuitry to compensate it, it is enough to choose a schmitt trigger with a lower threshold voltage from the very beginning.

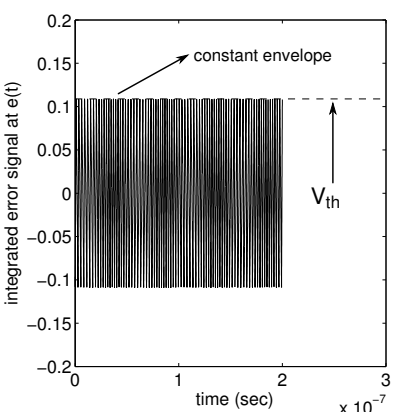

(a)

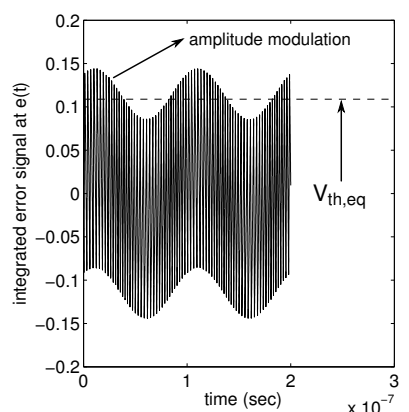

(b)
Fig. 3. typical waveforms of two ASDMs, (a) without any delay in the loop, and (b) with loop delay.

\section{C. an ASDM with a comparator and loop delay}

The theory presented in this work applies to ASDMs that use a schmitt trigger (with/without delay) as their nonlinear element. But the theory is still valid for the case that the threshold voltage of the schmitt trigger is zero and the loop delay is non-zero. In other words, it is possible to use (20) to design an ASDM with a comparator and some delay blocks (like digital buffers) which may be designed easier in comparison with a schmitt trigger.

\section{SIMULATION RESULTS}

In this section, we will describe a few simulations to confirm the proposed theory. In all cases, for every ASDM with loop delay, an equivalent ASDM without delay (with similar loop filter but higher threshold voltage according to section IV-A) has been simulated. Then the simulation results of these two ASDMs are compared with the proposed theory.

\section{A. output spectrum, first order loop filter}

In the first set of simulations, we will take a look at the output spectrum of two equivalent ASDMs. The schematic of the two ASDMs is according to Fig. 1 (a) and (b). They both have a first order passive loop filter, $F(s)=\frac{1}{1+\tau s}$, with $\tau=5 \mathrm{~ns}$. For the ASDM with delay, we have chosen $T_{D}=150 \mathrm{ps}$ and $V_{t h}=0.1$. Therefore, according to section IV-A, the equivalent delay-less ASDM should have a threshold voltage of $V_{t h, e q}=0.1266$.

Fig. 4 (a) and (b) show the output spectrum of these two ASDMs. According to [8] and (9) the average carrier frequency of an ASDM for a sinusoidal input voltage with an amplitude of $A$ is $\overline{\omega_{1}}=\omega_{c}\left(1-0.5 A^{2}\right)$. For these cases $A=0.25$ was chosen, so $f_{1}=380.5 \mathrm{MHz}$ is expected and according to (20), the predicted third harmonic distortion is $d_{3}=74.9 \mathrm{~dB}$. As the figure shows, there is good match between the two equivalant ASDMs and the proposed theory.

\section{B. a second order ASDM with comparator and loop delay}

In section IV-C we argued that an ASDM that has a comparator as its non-linear element (instead of a schmitt trigger) and some delay in its loop can be equivalent with a delay-less ASDM with schmitt trigger.

In order to verify this argument, for this example we have chosen a second order loop filter, $F(s)=\frac{1+s \tau_{1}}{s \tau_{2} \cdot s \tau_{3}}$, with 


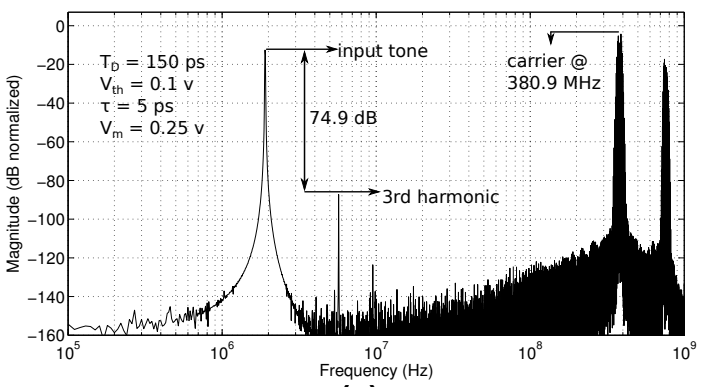

(a)

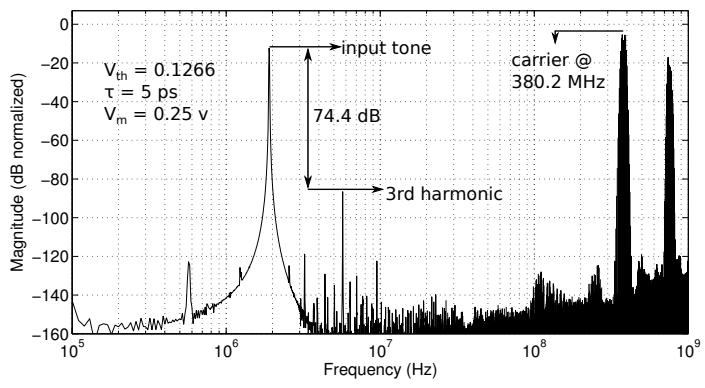

(b)

Fig. 4. Output spectrum of two equivalent ASDMs with first order loop filter, (a) with some delay in the loop, and (b) without loop delay.

$\tau_{1}=\tau_{2}=\tau_{3}=5 \mathrm{~ns}$. The first modulator uses a comparator in its loop $\left(V_{t h}=0\right)$ and has a delay of $T_{D}=600 \mathrm{ps}$. According to section IV-A, this modulator should be equivalent with another ASDM that uses a schmitt trigger with a threshold voltage of $V_{t h, e q}=0.1264$ and no delay, $T_{D}=0$. According to (9) These values correspond to a self oscillation (zero input) carrier frequency of $f_{c}=390 \mathrm{MHz}$

In Fig. 5(a) these two ASDMs have been compared with the theory in (20). A sinusoidal voltage with a frequency of $\mu=9.75 \mathrm{MHz}$ has been been applied to the input of both modulators and their third harmonic distortion $d_{3}$ has been evaluated over a range of input amplitudes. As the figure verifies, the two ASDMs are actually equivalent regarding their distortion and they both match well with the proposed theory in (20). A similar set of simulations have been carried out in Fig. 5 (b) where the same ASDMs have been applied a sinusoidal signal as their input voltage. This time the amplitude of the input is constant, $A=0.5$, and the frequency is swept. Once again a good match between the two modulators and theory can be seen.

\section{CONCLUSION}

We have derived Eq. (20) to predict the 3rd harmonic distortion of an ASDM with loop delay. We have also argued that an ASDM with loop delay is equivalent with a similar ASDM that has no delay, but a higher threshold voltage in its schmitt trigger. The simulation results matched very well with the analytical results and verified this equivalency.

From a designer's point of view, the main conclusion is that the distortion is essentially determined by the loop filter in combination with the self oscillation frequency. The delay in the loop affects the self oscillation frequency, but if the delay is known, this effect can be counter-acted by tuning the schmitt trigger's threshold voltage and choosing a lower $V_{t h}$.

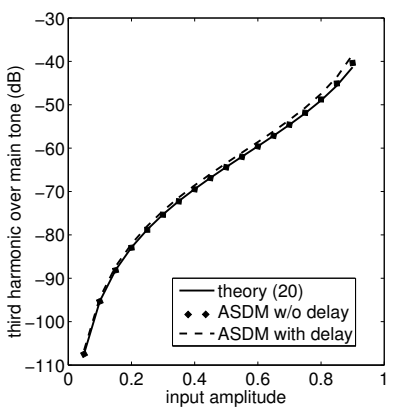

(a)

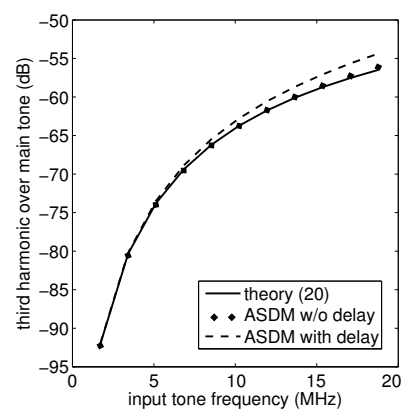

(b)
Fig. 5. Comparing the distortion of two equivalent second order ASDMs with theory.

From another perspective, for a given loop filter, better linearity can be obtained by increasing the oscillation frequency, $f_{c}$, which can be realized by decreasing the delay or lowering the threshold voltage of the schmitt trigger (even as low as zero). The price of this approach is of course higher switching activity and power consumption.

In practice, all of the relevant requirements can be optimized using the explicit equations that have been presented in this paper.

\section{ACKNOWLEDGEMENT}

This work has been supported by the Fund for Scientific Research Flanders (FWO-Vlaanderen) Belgium.

\section{REFERENCES}

[1] L. Hernandez, S. Paton, E. Prefasi, "VCO-based sigma delta modulator with PWM precoding," Electron. Lett., vol. 49, no. 10 pp. 558-559, 12th May 2011.

[2] G.W. Roberts and M. Ali-Bakhshian, "A Brief Introduction to Time-toDigital and Digital-to-Time Converters," IEEE Trans. Circuits Syst.-II, vol. 57 , no. 3, pp. 153-157, Mar. 2010.

[3] B. De Vuyst and P. Rombouts, "A 5-MHz 11-Bit Self-Oscillating Sigma Delta Modulator With a Delay-Based Phase Shifter in $0.025 \mathrm{~mm}^{2}$," IEEE J. Solid-State Circuits, vol. 46, no. 8, pp. 1919-1927, Aug. 2011.

[4] J. Daniels, W. Dehaene, M. S. J. Steyaert, and A. Wiesbauer, "A/D Conversion Using Asynchronous Delta-Sigma Modulation and Timeto-Digital Conversion," IEEE Trans. Circuits Syst.-I, vol. 57, no. 9, pp. 2404-2410, Sep. 2010.

[5] A. Babaie Fishani and P. Rombouts, "Continuous time $\Delta \Sigma$ modulation with PWM pre-coding and binary $g_{m}$ blocks," Electron. Lett., vol. 48, no. 19, pp. 1187-1188, Sep. 2012.

[6] M. Berkhout, "Class-D audio amplifiers in mobile applications," IEEE J. Solid-State Circuits, vol. 46, no. 8, pp. 1919-1927, Aug. 2011.

[7] T. Piessens and M.S.J. Steyaert, "Behavioral analysis of self-oscillating class D line drivers," IEEE Trans. Circuits Syst.-I, vol. 52, no. 4, pp. 706-714, Apr. 2005.

[8] A. Babaie-Fishani, B. Van Keymeulen, and P. Rombouts, "Analytical Expressions for the Distortion of Asynchronous Sigma-Delta Modulators," IEEE Trans. Circuits Syst.-II: Express Briefs, vol. 60, no. 8, pp. 472-476, 2013.

[9] E. Roza, "Analog-to-digital conversion via duty-cycle modulation," IEEE Trans. Circuits Syst.-II, vol. 44, no. 11, pp. 907-914, Nov. 1997.

[10] T. Matic, T.Sandvedek, and M. Herceg, "A Method for the SchmittTrigger Propagation-Delay Compensation in Asynchronous SigmaDelta Modulator," IEEE Trans. Circuits Syst.-II, vol. 59, no. 7, pp. 404-408, Jul. 2012.

[11] V. Mangulis, Handbook of series for scientists and engineers. New York: Academic, 1965. 